\section{VEGF-Hemmung bei EGFR-Wildtyp besser wirksam}

Die aktuelle S3-Leitlinie zu Diagnose und Therapie des Bronchialkarzinoms empfiehlt für die Erstlinientherapie den Einsatz des VEGF-Antikörpers Bevacizumab (Avastin ${ }^{\oplus}$ ) in Kombination mit einer platinbasierten Chemotherapie bei Patienten mit Nicht-Plattenepithelkarzinom im Stadium IIIB/ IV und gutem Allgemeinzustand (ECOG-Performance-Status 0 oder 1 ) [Goeckenjan G et al. Pneumologie. 2010;64(Suppl 2):S23-S155]. Eine in deutschen Zentren durch die ABCStudiengruppe durchgeführte randomisierte Phase-II-Studie „INNOVATIONS“verglich eine chemotherapiefreie Kombination aus Bevacizumab plus dem EGFR-TyrosinkinaseInhibitor Erlotinib mit der Kombination von Bevacizumab mit Cisplatin/ Gemcitabin in einem unselektionierten Patientenkollektiv mit neu diagnostiziertem, nicht-squamösem NSCLC im Stadium IIIB/IV [Thomas M et al. J Clin Oncol. 2011;29:15s(Suppl) Abstr 7504]. Dabei war die chemotherapiefreie Kombination nicht überlegen und die Wirksamkeit der Kombination von Cisplatin/Gemcitabin mit Bevacizumab wurde erneut deutlich: Die Auswertung des unselektionierten Gesamtkollektivs ergab für den chemotherapiehaltigen Arm ( $n=113)$ ein medianes progressionsfreies Überleben (PFS) von 7,7 Monaten und ein medianes Gesamtüberleben (OS) von 16,3 Monaten. Von 161 der 224 an der Studie teinehmenden Patienten war auch der EGFR-Mutationsstatus bekannt. Patienten ohne nachgewiesene EGFR-Mutation profitierten nach den Analysen besonders von der Bevacizumab-Zytostatika-Kombination: Sie erreichten ein medianes PFS von 8,4 Monaten (95\%-Konfidenzintervall [95\%-KI]: 6,7-9,7 Monate) und ein medianes OS von 18,0 Monaten (95\%KI: 12,0 Monate-nicht erreicht). Die Ansprechrate lag im Gesamtkollektiv bei $37 \%$ und bei der Subgruppe der Patienten ohne EGFR-Mutation bei $41 \%$.

Nach Informationen von Roche

Pankreatische Neuroendokrine Tumoren

\title{
Zielgerichtet gegen seltene Tumoren
}

\author{
Pankreatische Neuroendokrine Tumoren (pNET) zeigen \\ häufig ein langsames Wachstum, nehmen aber letztlich \\ eine maligne Entwicklung. Patienten mit fortgeschritte- \\ nen, progredienten pNET können von einer Behandlung \\ mit Tyrosinkinase-Inhibitoren (TKI) profitieren.
}

Der Multi-TKI Sunitinib (Sutent ${ }^{\circledR}$ ), beim metastasierten Nierenzellkarzinom bzw. bei Gastrointestinalen Stromatumoren (GIST) bereits ein Standard, wurde 2010 für die Behandlung progredienter, kurativ nicht resezierbarer, gut differenzierter pankreatischer NET (pNET G1 bzw. G2) zugelassen, berichtete Ulrich-Frank Pape, Berlin. Die Zulassung basiert auf einer Phase-III-Studie, in der die Wirksamkeit und Sicherheit von 37,5 mg/Tag Sunitinib randomisiert und doppelblind mit Placebo verglichen wurden.

Die Studie wurde vorzeitig abgebrochen, nachdem eine Interimsanalyse eine klare Überlegenheit von Sunitinib mit einer Verdopplung des progressionsfreien Überle- bens auf 11,4 vs. 5,5 Monate (HR 0,42, p < $0,001)$ ergeben hatte [Raymond $\mathrm{E}$ et al. $\mathrm{N}$ Engl J Med. 2011;364(6):501-13]. Dabei profitierten alle analysierten Subgruppen von Sunitinib. Außerdem war der TKI Sunitinib mit einer deutlichen Reduktion der Gesamtmortalität (OS) assoziiert (HR 0,41, $\mathrm{p}=0,02)$. Die mediane Verlängerung des OS durch Sunitinib betrug 6,1 Monate (30,5 vs. 24,4 Monate) [Valle J et al. ECCO/ ESMO. 2011; Abstract 6569].

Günter Springer

Expertengespräch „Zielgerichtete Therapie bei pankreatischen NET (pNET): Worauf kommt es an?" am 7. März 2012 in Kopenhagen anlässlich des ENETS 2012; Veranstalter: Pfizer.

\section{Antikoagulation}

\section{Thromboembolierisiko verringern}

\author{
Mit den neuen oralen Antithrombosepräparaten hat sich \\ in vielen Feldern der Antikoagulation ein Paradigmen- \\ wechsel vollzogen - aber nicht in allen.
}

Patienten mit Tumorerkrankung haben ein vier- bis siebenfach erhöhtes Risiko für das Auftreten einer venösen Thromboembolie (VTE). Ohne Prophylaxe beträgt die Inzidenz der tiefen Venenthrombose (TVT) nach Tumoroperation 40-80\% (proximale TVT: 10-20\%) und das Risiko einer fatalen Lungenembolie vergrößert sich um das Vierfache.

Ulrich Hoffmann, München, verwies auf die Leitlinien des American College of Clinical Pharmacy (ACCP), die für Tumorpatienten mit hohem Risiko für eine VTE, aber ohne erhöhtes Blutungsrisiko, eine vierwöchige Prophylaxe mit niedermolekularen Heparinen (NMH) empfehlen [Guyatt GH et al. Chest. 2012;141(2 Suppl): 7S-47S]. Durch diese Maßnahme können die Inzidenz der TVT um $50 \%$ und die der proximalen TVT um $75 \%$ gesenkt werden.
Susanne Alban, Kiel, ergänzte, dass NMH wie Tinzaparin (Innohep ${ }^{\circledR}$ ) keine pharmakokinetischen Interaktionen und keine toxischen Effekte auslösen. Die parenterale Applikation garantierte eine sichere Bioverfügbarkeit. Zudem handele es sich um multivalente Biomodulatoren, die durch antiinflammatorische, komplementhemmende und antimetastatische und antiangiogene Effekte einen Beitrag zur therapeutischen Wirksamkeit leisten - Zusatzeffekte, die laut Alban von selektiven Inhibitoren nicht zu erwarten seien.

Ine Schmale

Symposium „Mehr als Antikoagulation - Die Bedeutung niedermolekularer Heparine in der Tumortherapie" am 10. Mai 2012 in Mainz anlässlich des 37. Kongresses des Bundesverbandes Deutscher Krankenhausapotheker (ADKA) ;

Veranstalter: LEO. 